\title{
Targeted deep amplicon sequencing of kelch 13 and cytochrome $b$ in Plasmodium falciparum isolates from an endemic African country using the Malaria Resistance Surveillance (MaRS) protocol
}

Mariangela L'Episcopia1*, Julia Kelley², Dhruviben Patel², Sarah Schmedes³, Shashidahar Ravishankar4, Michela Menegon', Edvige Perrotti ${ }^{1}$, Abduselam M. Nurahmed ${ }^{5}$, Albadawi A. Talha ${ }^{6,7}$, Bakri Y. Nour ${ }^{8}$, Naomi Lucchi ${ }^{9}$, Carlo Severini ${ }^{1}$ and Eldin Talundzic ${ }^{9}$

\begin{abstract}
Background: Routine molecular surveillance for imported drug-resistant malaria parasites to the USA and European Union is an important public health activity. The obtained molecular data are used to help keep chemoprophylaxis and treatment guidelines up to date for persons traveling to malaria endemic countries. Recent advances in next-generation sequencing (NGS) technologies provide a new and effective way of tracking malaria drug-resistant parasites.

Methods: As part of a technology transfer arrangement between the CDC Malaria Branch and the Istituto Superiore di Sanità (ISS), Rome, Italy, the recently described Malaria Resistance Surveillance (MaRS) protocol was used to genotype 148 Plasmodium falciparum isolates from Eritrea for kelch 13 (k13) and cytochrome b (cytb) genes, molecular markers associated with resistance to artemisinin (ART) and atovaquone/proguanil (AP), respectively.

Results: Spanning the full-length $k 13$ gene, seven non-synonymous single nucleotide polymorphisms (SNPs) were found (K189N, K189T, E208K, D281V, E401Q, R622I and T535M), of which none have been associated with artemisinin resistance. No mutations were found in cytochrome $b$.

Conclusion: All patients successfully genotyped carried parasites susceptible to ART and AP treatment. Future studies between CDC Malaria Branch and ISS are planned to expand the MaRS system, including data sharing, in an effort to maintain up to date treatment guidelines for travelers to malaria endemic countries.
\end{abstract}

Keywords: Plasmodium falciparum, Drug resistance, Next-generation sequencing, Molecular surveillance

*Correspondence: mariangela.lepiscopia@iss.it

1 Department of Infectious Diseases, Istituto Superiore di Sanità, Rome, Italy

Full list of author information is available at the end of the article

(c) The Author(s) 2020. This article is licensed under a Creative Commons Attribution 4.0 International License, which permits use, sharing, adaptation, distribution and reproduction in any medium or format, as long as you give appropriate credit to the original author(s) and the source, provide a link to the Creative Commons licence, and indicate if changes were made. The images or other third party material in this article are included in the article's Creative Commons licence, unless indicated otherwise in a credit line to the material. If material is not included in the article's Creative Commons licence and your intended use is not permitted by statutory regulation or exceeds the permitted use, you will need to obtain permission directly from the copyright holder. To view a copy of this licence, visit http://creativeco mmons.org/licenses/by/4.0/. The Creative Commons Public Domain Dedication waiver (http://creativecommons.org/publicdomain/ zero/1.0/) applies to the data made available in this article, unless otherwise stated in a credit line to the data. 


\section{Background}

In the last decade, total malaria cases have been reduced by $40 \%$ worldwide $[1,2]$ leading to a dramatic reduction in mortality in children, especially in subSaharan Africa. This was achieved through the deployments of vector control measures, accurate diagnosis, and treatment of uncomplicated Plasmodium falciparum malaria with artemisinin-based combination therapies (ACTs). Despite this overall progress, thousands of travel-related malaria cases are imported into the European Union (EU) and the USA [3, 4]. The European Centre for Disease Prevention and Control (ECDC) estimate that an average of 8000 malaria cases are imported to the EU annually, most of which are travelers returning home after visiting friends and relatives in Africa [4]. In the USA, according to the Centers for Disease Control and Prevention (CDC), approximately 1700 malaria cases are imported annually to the country [3].

Both the EU and the USA, recommend the use of atovaquone/proguanil (AP) for chemoprophylaxis [5-7]. In the USA, AP is used as a primary treatment choice for imported uncomplicated $P$. falciparum cases, while in the EU, two ACTs, artemether-lumefantrine (AL) and dihydroartemisinin/piperaquine (DHA/PPQ) are licensed as treatment options for uncomplicated $P$. falciparum malaria [8-10]; AP can be used if an ACT is not available [10].

Single nucleotide polymorphisms (SNPs) in the cytochrome $b(c y t b)$ gene, in the mitochondrial genome of $P$. falciparum, confer resistance to AP. The putative I258M and Y268S/C SNPs have been associated with AP resistance $[11,12]$. In comparison, resistance to artemisinin derivatives, given as part of ACTs, such as AL, are reported with parasites carrying SNPs in the kelch 13 (k13) gene. Specifically, the F446I, N458Y, M476I, Y493H, R539T, I543T, P553L, R561H and C580Y SNPs are confirmed to confer resistance to artemisinin. [13]. The $k 13$ gene provides a validated molecular marker to detect, track and monitor the emergence and spread of artemisinin-resistant SNPs [14-16]. While a number of other $k 13$ SNPs have been described in Africa, none have yet been confirmed to be associated with artemisinin resistance [17-19], except in a case described by Lu et al. [20] in which was shown that a P. falciparum imported into China from Equatorial Guinea was resistant to artemisinin in vitro.

Molecular surveillance of imported malaria cases is an important public health activity that can aid in the detection of drug-resistant $P$. falciparum parasites [21, 22]. Recent advancements in next-generation sequencing are providing a new way to rapidly detect and characterize drug-resistant malaria parasites, including minor parasite populations in mixed infection P. falciparum cases [23, 24]. These molecular data can be used to help keep chemoprophylaxis and treatment guidelines up to date for persons traveling to malaria endemic countries.

Towards this end, as part of a technology transfer training agreement between the CDC and the Istituto Superiore di Sanità, Rome, Italy, we used the recently published Malaria Resistance Surveillance (MaRS) [24] protocol to genotype SNPs in the kelch 13 and cytochrome $b$ genes from 148 P. falciparum-positive samples from Eritrea.

\section{Methods \\ Samples}

A total of 148 P. falciparum dried blood spots (DBS) were used in this study; these were originally collected as part of a cross-sectional study between November 2013 and November 2014 in two regions (Barentu and Agordat) of Eritrea endemic for falciparum malaria, as previously described by Menegon et al. [25]. Samples were initially analyzed for $P$. falciparum infection using molecular diagnosis at the Gezira University (Wad Medani, Sudan) in a collaboration existing between Gezira University and the Eritrean Ministry of Health.

\section{DNA extraction}

DNA was extracted using the QIAmp DNA Blood Mini Kit (Qiagen, Valencia, CA, USA) using the recommended guidelines in the original MaRS protocol [24].

\section{Plasmodium falciparum species confirmation and sample quality assurance}

The previously described real-time PET-PCR method [26] was used to confirm $P$. falciparum infections and assess the quality of DNA from the filter blood spot samples. This method is based on self-quenching photo-induced electron transfer (PET) fluorogenic primers used in a multiplex real-time PCR to detect Plasmodium spp. and $P$. falciparum. Samples with a Cq value $>40$ were considered $P$. falciparum-negative.

\section{Kelch 13 and cytochrome $b$ PCR enrichment}

The following primers were used: $k 13$ gene (forward: 5'-CTA TGA CGT ATG ATA GGG AAT CTG G-3' and reverse: $5^{\prime}$-CTG GGA ACT AAT AAA GAT GGG CC-3'); cytochrome $b$ gene (forward: $5^{\prime}$-CTA TTA ATT TAG TTA AAG CAC AC- $3^{\prime}$ and reverse: $5^{\prime}$-ACA GAA TAA TCT CTA GCA CCA-3'). Previously described PCR conditions were used [24]. Plasmodium falciparum kelch13 wild-type strains HB3, 7G8 and P. falciparum kelch13 C580Y mutant strain MRA1236 (ATCC) were used as controls. DNA isolated from whole blood 
of individuals without malaria was used as a negative control.

\section{PCR product purification, normalization, pooling, library prep and sequencing}

Briefly, PCR products were purified and normalized using the SequalPrep Kit (Cat \# A1051001; Thermo Fisher Scientific, Waltham, USA). PCR products were pooled in $10.0 \mu \mathrm{l}$ volumes for each sample. Library prep was performed using the Illumina Nextera XT kit (Cat \# FC-131-1096 and FC-131-1002; Illumina, San Diego, USA). Pooled fragments were assessed for correct size using the Agilent D5000 ScreenTape Station (Cat \# G2940C, \# 5067-4626, and \# G2940CA; Agilent Technologies, Santa Clara, USA) and DNA concentration checked using the Qubit 3.0 Flurometer (Cat \#Q33216 and \#Q32853; Life Technologies Corporation, Carlsbad, USA). Sequencing was performed using the MiSeq v2 reagents using the 500 cycle kit (Cat \# MS-102-2003; Illumina MiSeq reagents v2, San Diego, USA).

\section{Data analysis}

The next-generation sequencing analysis toolkit (NeST) (https://github.com/CDCgov/MaRS) was used to call non-synonymous and synonymous single nucleotide polymorphisms (SNPs) in $k 13$ and cytb genes, respectively. All SNPs were also confirmed using the Geneious Prime software (www.geneious.com).

\section{MaRS protocol details}

Details about the laboratory and data analysis protocols can be found at https:/github.com/CDCgov/ MaRS. This protocol is based on collating and mapping SNPs associated with antimalarial drug resistance by using a targeted amplicon deep sequencing (TADS) approach.

\section{Results}

\section{Sequencing outcome using dried blood spots}

PET-PCR was performed on all 148 samples in duplicate. A total of 144 out of 148 screened samples were positive for both the genus Plasmodium and P. falciparum based on the Cq values (mean of 30.9). Out of the positive samples, successful sequencing results were obtained for $82.6 \%(119 / 144)$ and $91.7 \%(132 / 144)$ samples for the $k 13$ and $c y t b$ genes, respectively, as shown in Fig. 1.

\section{Read depth for SNPs associated with malarial drug-resistant $k 13$ and cytb}

Read depth at 2 SNPs in the $c y t b$ and 21 SNPs (9 validated SNPs and 12 candidate SNPs based on the 2018 WHO artemisinin report) in $k 13$ for 119 and 132 samples, respectively, were analyzed (Fig. 2). Median read depth across SNPs in $c y t b$ was $310.5 \times$ coverage. The median read depth across SNPs in $k 13$ was 126X coverage.

\section{Kelch 13}

Spanning the full-length $k 13$ gene in the $82.6 \%$ $(119 / 144)$ successfully sequenced samples, seven nonsynonymous SNPs were detected (Table 1). Of the seven non-synonymous mutations detected, five were found outside the propeller domain region: K189N, K189T, E208K, D281V and E401Q. Of these, the most frequent SNP was K189T found in 59.7\% (71/119) of samples at an allele frequency of $100 \%$, except for one sample in which the polymorphism was identified as minor allele at an allele frequency of $43.0 \%$. Inside the $k 13$ propeller domain, two non-synonymous SNPs were identified: R622I and T535M. R622I was detected as a major allele at an allele frequency of $100 \%$ and T535M as a minor allele at an allele frequency of less of $50 \%$ (Table 1).

\section{Cytochrome $b$}

A total of $91.7 \%(132 / 144)$ were successfully sequenced for $c y t b$. Of these, 98.5\% (130/132) carried the wildtype $258 \mathrm{I}$ and $268 \mathrm{Y}$ alleles. Two samples were identified with the V127V synonymous mutation (Table 1).

\section{Discussion}

Active surveillance of imported malaria cases is an important public health activity in the USA and the EU $[27,28]$ : it plays a pivotal role in estimating the incidence in imported malaria cases, preventing malaria reintroduction, and providing chemoprophylaxis guidelines to travelers to malaria endemic regions. Historically, anti-malaria drug resistance genotyping was performed using traditional low throughput sequencing methodologies, such as Sanger sequencing [29]. However, currently, next-generation sequencing and accompanying standardized bioinformatics tools provide advanced and rapid protocols for monitoring molecular marker genes involved in $P$. falciparum drug resistance [24].

Based on the WHO guidelines, routine malaria surveillance is critical for the intervention in all malaria endemic and non-endemic countries, with the goal to reduce the incidence of malaria, eliminate the disease and prevent its re-establishment through detection and characterization of malaria parasites [30]. To achieve this goal, laboratory and analysis protocols related to malaria molecular surveillance activities need to be standardized, have appropriate quality assurance systems in place, and provide information 

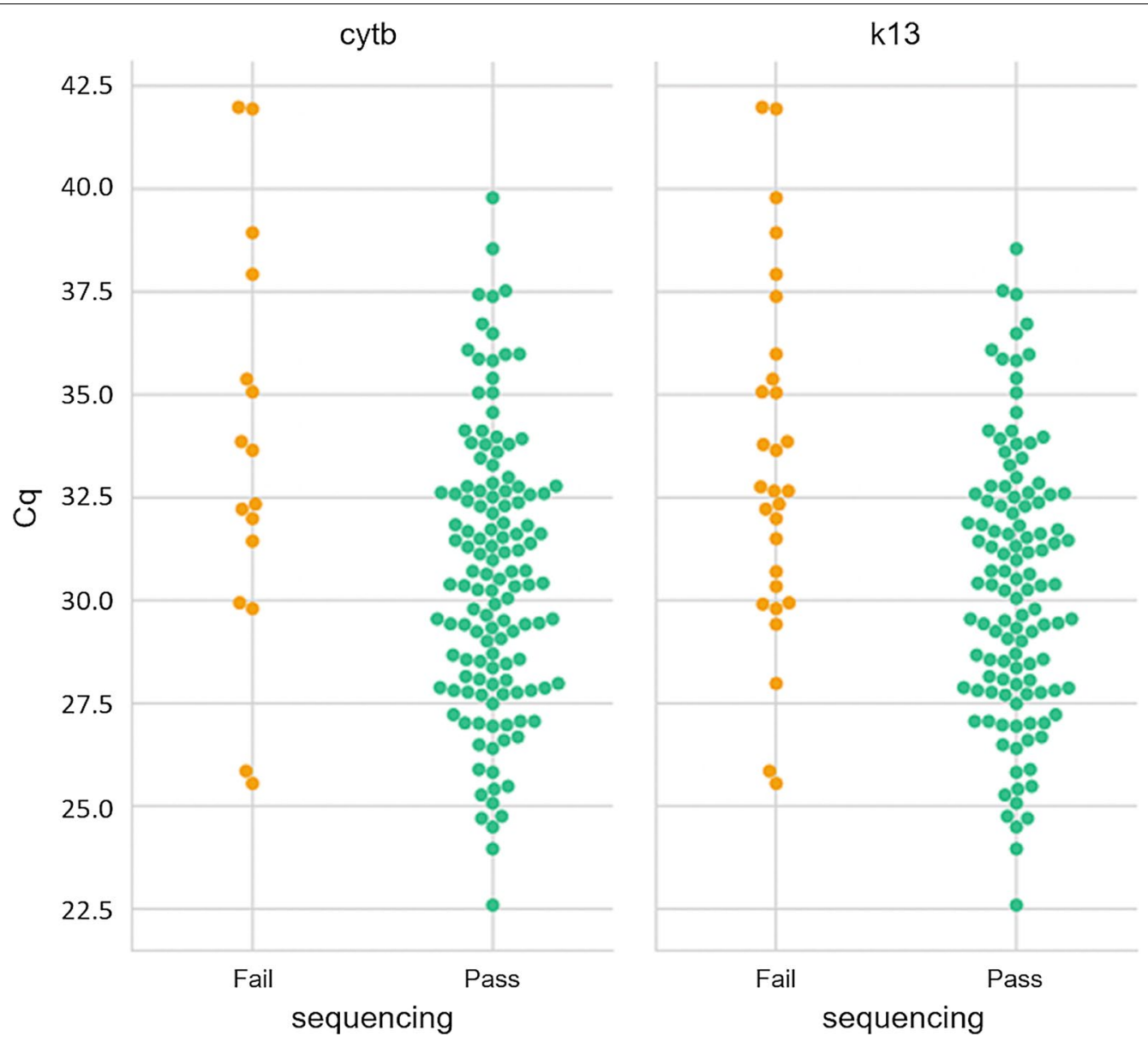

Fig. 1 Sequencing outcome for kelch 13 and cytochrome $b$ genes. Sequencing pass (green dots) or fail (red dots) is shown on the $x$-axis and Cq values for each sample on the $y$-axis. Successful sequencing data were obtained for $82.6 \%(119 / 144)$ and $91.7 \%(132 / 144)$ samples for the $k 13$ and cytb genes, respectively

in a timely fashion. The recent advances in next-generation sequencing (NGS), including high throughput and decreasing costs, meet these requirements and are making this technology more suitable for routine molecular surveillance of $P$. falciparum drug resistance genes in public health laboratories [24]. Notably, with this approach, lots of samples can be analyzed simultaneously, allow focusing on the full length of the genes, rather than only some regions. Toward this end, as part of a technology transfer arrangement between the CDC and the Istituto Superiore di Sanità, Rome, the recently developed Malaria Resistance Surveillance (MaRS) protocol was used to genotype $P f k 13$ and $P f c y t b$ genes using dried blood spot samples available for the present study. The $k 13$ and $c y t b$ genes were genotyped since AP, $\mathrm{AL}$ and DHA/PPQ are used for treatment of malaria cases imported into Italy.

Our results are in agreement with previously published reports on the $k 13$ gene from Africa, where a large number of non-artemisinin resistant associated SNPs have been reported. Notably, the K189T mutation, which is widely prevalent in African artemisininsensitive $P$. falciparum parasites [31] as well as in South East Asian parasites [32] was observed. We found no evidence of the putative I258M and Y268S/C SNPs associated with AP resistance $[11,12]$, suggesting that the use of AP for prophylaxis for travelers to Eritrea remains effective.

\section{Conclusions}

The MaRS protocol used in this technology transfer training provides a standardized, high-throughput laboratory and analysis system for characterizing and tracking possible polymorphisms in molecular marker genes linked to drug resistance. The goal of this study was to evaluate the possibility to set up MaRS system also in Italy and to maintain active collaboration between the two institutes. 


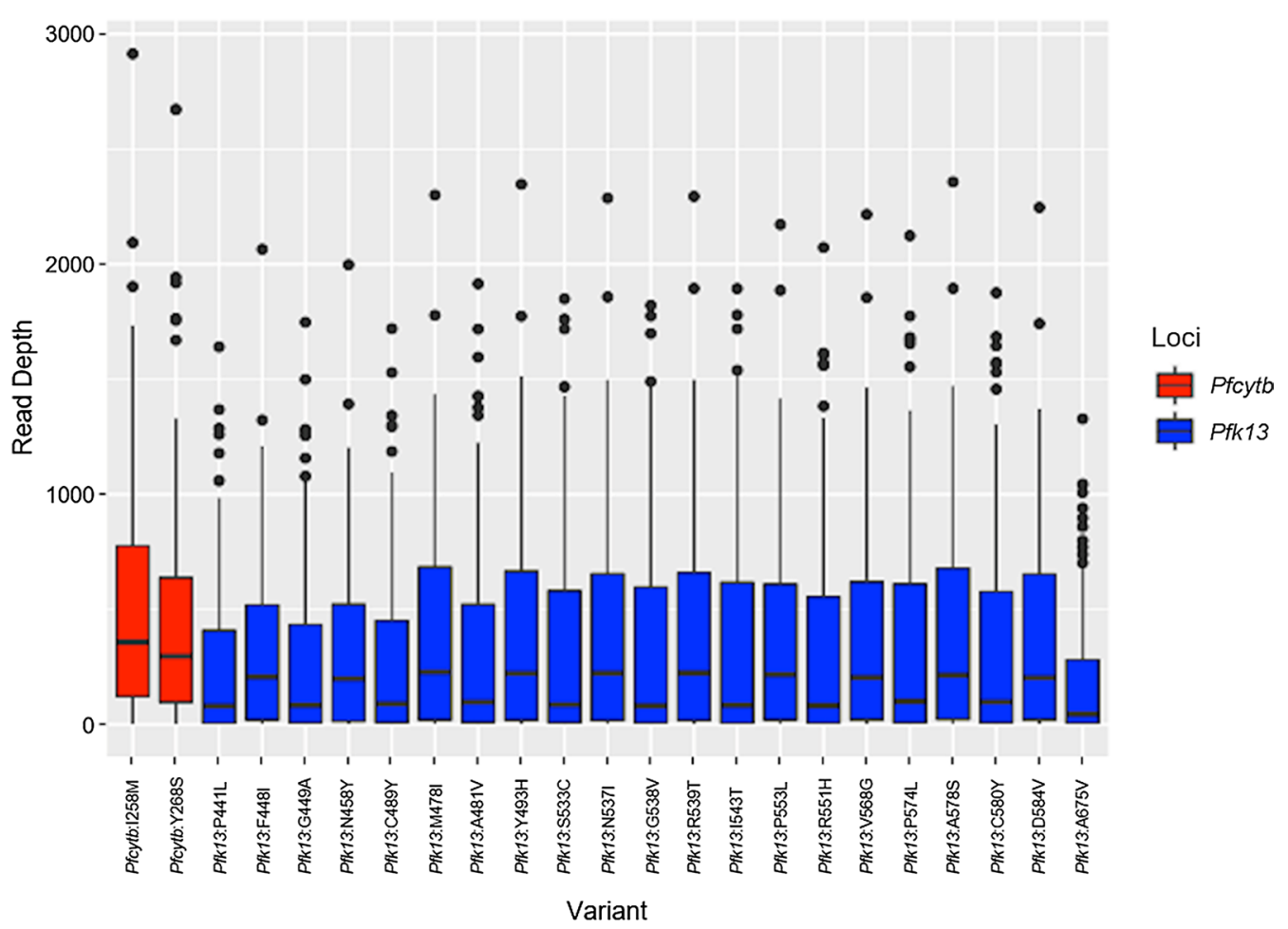

Fig. 2 Read depth for SNPs associated with malarial drug resistance in the Plasmodium falciparum cytb and k13. Middle bar, median; upper box hinge, 75th percentile; lower box hinge, 25th percentile; upper whisker, largest value no further than $1.5 \times 1 \mathrm{IQR}$ (inter-quartile range or distance from first and third quartiles) from the hinge; lower whisker, smallest value at most $1.5 \times 1 Q R$ from the hinge; dots, outlying samples

Table 1 Prevalence of polymorphisms in kelch 13 and cytochrome $b$ genes in the analyzed falciparum isolates. K13 protein consists of a Plasmodium-specific N-terminal region (Pf N-t region), coiled-coil-containing domain (CCC), a Broad-complex, Tramtrack, Bric-a-Brac/ Poxvirus and Zincfinger domain (BTB/POZ) and a C-terminal Kelch-repeat propeller domain (KREP)

\begin{tabular}{|c|c|c|c|c|c|c|c|c|c|}
\hline \multirow[t]{2}{*}{ Target gene } & \multirow[t]{2}{*}{ Codon position } & \multirow[t]{2}{*}{ Domain location } & \multicolumn{2}{|c|}{ Reference sequence } & \multicolumn{2}{|c|}{ Mutant sequence } & \multirow[t]{2}{*}{ Type } & \multirow{2}{*}{$\begin{array}{l}\text { Allele } \\
\text { frequency } \\
(\%)\end{array}$} & \multirow[t]{2}{*}{$n / N$} \\
\hline & & & Amino acid & Nucleotide & Amino acid & Nucleotide & & & \\
\hline \multirow[t]{8}{*}{ Pfk 13} & 189 & Pf $\mathrm{N}$-t region & K & AAA & N & AAT & NS & 100 & $4 / 119$ \\
\hline & 189 & Pf $\mathrm{N}$-t region & K & $\mathrm{AAA}$ & $\mathrm{T}$ & ACA & NS & 100 & $70 / 119$ \\
\hline & 189 & Pf $\mathrm{N}$-t region & K & $\mathrm{AAA}$ & $\mathrm{T}$ & $\mathrm{ACA}$ & NS & 43 & $1 / 119$ \\
\hline & 208 & Pf $\mathrm{N}$-t region & $\mathrm{E}$ & GAA & K & AAA & NS & 100 & 10/119 \\
\hline & 281 & $\mathrm{CCC}$ & $\mathrm{D}$ & GAT & V & $\mathrm{GTT}$ & NS & 100 & $2 / 119$ \\
\hline & 401 & BTB/POZ & E & GAG & Q & CAG & NS & 100 & $4 / 119$ \\
\hline & 622 & KREP & $\mathrm{R}$ & AGA & । & ATA & NS & 100 & $1 / 119$ \\
\hline & 535 & KREP & $\mathrm{T}$ & $\mathrm{ACG}$ & M & ATG & NS & 20 & $1 / 119$ \\
\hline Pfcytb & 127 & - & V & GTG & V & GTG & Syn & 100 & $2 / 132$ \\
\hline
\end{tabular}

Note: The boldface highlights the nucleotide base change

Abbreviations: $\mathrm{n}$, number of samples containing mutant allele; $\mathrm{N}$, total number of successfully sequenced samples; NS, non-synonymous mutation

\section{Abbreviations}

NGS: next-generation sequencing; CDC: Centers for Disease Control and Prevention; ISS: Istituto Superiore di Sanità; MaRS: Malaria Resistance Surveillance; k13: kelch 13 gene; cytb: cytochrome b gene; ART: artemisinin; ATQ: atovaquone; AP: atovaquone/proguanil; SNPs: single nucleotide polymorphisms; ACT: artemisinin-based combination therapy; ECDC: European Centers for Disease
Control and Prevention; AL: artemether/lumefantrine; DHA: dihydroartemisinin; PPQ: piperaquine; DBS: dried blood spot; PET: photo-induced electron transfer; PCR: polymerase chain reaction; NeST: next-generation sequencing analysis toolkit; IQR: inter-quartile range.

\section{Acknowledgments}

We thank Peter McElroy, Michael Aidoo and Venkatachalam Udhayakumar for reviewing this manuscript and we want to thank Barbara J. Marston for 
reviewing and approving this study under the human subject's protocol 2017-228.

\section{Authors' contributions}

$\mathrm{ML}, \mathrm{CS}$ and ET designed the study and wrote the manuscript. ML and JK performed the experiments. ML, DP, SS, SR and ET performed the data analysis and figure generation. MM, EP, AN, AT and BN provided the samples, reviewed and approved the study. NL contributed to initiating this collaboration and assisted with editing of the final manuscript. All authors read and approved the final manuscript.

\section{Funding}

This study was made possible through support from the Advanced Molecular Detection (AMD) initiative at the CDC. We also acknowledge partial support by the Bioinformatics Fellowship Program administered by the Association of Public Health Laboratories (APHL) and funded by the CDC. SES is currently supported in part by the Bioinformatics Fellowship Program administered by the APHL and funded by the CDC. JK is currently supported in part by the CDC Foundation. DP is employed by Williams Consulting LLC. Williams Consulting LLC provided support in the form of salary for DP. The funders had no role in study design, data collection and analysis, decision to publish, or preparation of the manuscript. The specific role of this author is articulated in the 'Authors' contributions' section.

\section{Availability of data and materials}

The datasets generated during the present study are available in the PRJNA428490 repository, https://www.ncbi.n/m.nih.gov/Traces/study $/$ acC $=$ SRP128900

\section{Ethics approval and consent to participate}

Original ethical approval for drug resistance studies was obtained from the Ethics Committee of the Eritrean Ministry of Health (OSM 29/377/13). The present study was approved by the Office of the Associate Director of Science, Center for Global Health, Center for Disease Control and Prevention as research not involving human subjects (protocol 2017-228). In May 2017, the Department of Infectious Diseases, Istituto Superiore di Sanità, Rome, Italy; the Malaria Branch laboratory at the Centers for Disease Control and Prevention (CDC), Atlanta, USA and the University of Gezira, Wad Medani, Sudan agreed to work together on generating molecular data related to the original study entitled "Genetic basis of malaria drug resistance in Eritrea". For all samples, identifying information was removed so that data cannot be linked or relinked with identifiable human subjects.

\section{Consent for publication}

Not applicable.

\section{Competing interests}

The authors declare that they have no competing interests.

\section{Author details}

${ }^{1}$ Department of Infectious Diseases, Istituto Superiore di Sanità, Rome, Italy.

${ }^{2}$ Atlanta Research and Education Foundation, VAMC, Atlanta, Georgia, USA.

${ }^{3}$ Association of Public Health Laboratories, Silver Spring, MD, USA. ${ }^{4}$ School of Biology, Georgia Institute of Technology, Atlanta, Georgia, USA. ${ }^{5}$ Cansford Laboratories, Pentwyn Business Centre, Cardiff, UK. ${ }^{6}$ Faculty of Medical Laboratory Science, University of Gezira, Gezira, Sudan. ${ }^{7}$ Department of clinical laboratory Sciences, College of Applied Medical Sciences, Juof University, Sakaka, Saudi Arabia. ${ }^{8}$ Blue Nile Research National Institute for Communicable Diseases, University of Gezira, Wad Medani, Sudan. ${ }^{9}$ Centers for Disease Control and Prevention, CGH, DPDM, Atlanta, GA, USA.

Received: 21 November 2019 Accepted: 3 March 2020 Published online: 14 March 2020

\section{References}

1. Bhatt S, Weiss DJ, Cameron E, Bisanzio D, Mappin B, Dalrymple U, et al. The effect of malaria control on Plasmodium falciparum in Africa between 2000 and 2015. Nature. 2015;526:207-11.
2. WHO. World Malaria Report. 2018. Geneva: World Health Organization; 2018. https://www.who.int/malaria/publications/world-malaria-repor t-2018/report/en/.

3. Mace KE, Arguin PM, Tan KR. Malaria surveillance-United States, 2015. MMWR Surveill Summ. 2018;67:1-28.

4. ECDC. European Centre for Disease Prevention and Control. Annual epidemiological report for 2017. Surveillance report. Stockholm; 2019. https://www.ecdc.europa.eu/en/publications-data/malaria-annual-epide miological-report-2017.

5. CDC. Centers for Disease Control and Prevention: Malaria. Guidelines for Treatment of Malaria in the United States; 2015. https://www.cdc.gov/ malaria/resources/pdf/treatment_guidelines_101819.pdf.

6. LaRocque RC, Rao SR, Lee J, Ansdell V, Yates JA, Schwartz BS, et al. Global TravEpiNet: a national consortium of clinics providing care to international travelers - analysis of demographic characteristics, travel destinations, and pretravel healthcare of high-risk US international travelers, 2009-2011. Clin Infect Dis. 2012;54:455-62.

7. Shellvarajah M, Hatz C, Schlagenhauf P. Malaria prevention recommendations for risk groups visiting sub-Saharan Africa: a survey of European expert opinion and international recommendations. Travel Med Infect Dis. 2017;19:49-55.

8. Cordel H, Cailhol J, Matheron S, Bloch M, Godineau N, Consigny PH, et al. Atovaquone-proguanil in the treatment of imported uncomplicated Plasmodium falciparum malaria: a prospective observational study of 553 cases. Malar J. 2013;12:399.

9. WHO. Guidelines for the treatment of malaria. Geneva: World Health Organization; 2015. http://apps.who.int/medicinedocs/documents/s2183 9en/s21839en.pdf.

10. Lalloo DG, Shingadia D, Bell DJ, Beeching NJ, Whitty CJM, Chiodini PL, et al. UK malaria treatment guidelines 2016. J Infect. 2016;72:635-49.

11. Plucinski MM, Huber CS, Akinyi S, Dalton W, Eschete M, Grady K, et al. Novel mutation in cytochrome $b$ of Plasmodium falciparum in one of two atovaquone-proguanil treatment failures in travelers returning from same site in Nigeria. Open Forum Infect Dis. 2014;1:ofu059.

12. Kessl J, Ha KH, Merritt AK, Lange BB, Hill P, Meunier B, et al. Cytochrome b mutations that modify the ubiquinol-binding pocket of the cytochrome bc1 complex and confer anti-malarial drug resistance in Saccharomyces cerevisiae. J Biol Chem. 2005;280:17142-8.

13. WHO. Artemisinin resistance and artemisinin-based combination therapy efficacy. Geneva: World Health Organization; 2018. https://apps.who.int/ medicinedocs/documents/s23555en/s23555en.pdf.

14. Ariey F, Witkowski B, Amaratunga C, Beghain J, Langlois AC, Khim N, et al. A molecular marker of artemisinin-resistant Plasmodium falciparum malaria. Nature. 2014:505:50-5.

15. Takala-Harrison S, Jacob CG, Arze C, Cummings MP, Silva JC, Dondorp $\mathrm{AM}$, et al. Independent emergence of artemisinin resistance mutations among Plasmodium falciparum in Southeast Asia. J Infect Dis. 2015;211:670-9.

16. Mishra N, Prajapati SK, Kaitholia K, Bharti RS, Srivastava B, Phookan S, et al. Surveillance of artemisinin resistance in Plasmodium falciparum in India using the kelch13 molecular marker. Antimicrob Agents Chemother. 2015;59:2548-53.

17. Kamau E, Campino S, Amenga-Etego L, Drury E, Ishengoma D, Johnson K, et al. K13-propeller polymorphisms in Plasmodium falciparum parasites from sub-Saharan Africa. J Infect Dis. 2015;211:1352-5.

18. Taylor SM, Parobek CM, DeConti DK, Kayentao K, Coulibaly SO, Greenwood BM, et al. Absence of putative artemisinin resistance mutations among Plasmodium falciparum in sub-Saharan Africa: a molecular epidemiologic study. J Infect Dis. 2015;211:680-8.

19. Ménard D, Khim N, Beghain J, Adegnika AA, Shafiul-Alam M, Amodu O, et al. A Worldwide map of Plasmodium falciparum K13-propeller polymorphisms. N Engl J Med. 2016;374:2453-64.

20. Lu F, Culleton R, Zhang M, Ramaprasad A, von Seidlein L, Zhou H, et al. Emergence of indigenous artemisinin-resistant Plasmodium falciparum in Africa. N Engl J Med. 2017;376:991-3.

21. Russo G, L'Episcopia M, Menegon M, Souza SS, Dongho BGD, Vullo V, et al. Dihydroartemisinin-piperaquine treatment failure in uncomplicated Plasmodium falciparum malaria case imported from Ethiopia. Infection. 2018:46:867-70.

22. Tawe L, Menegon M, Ramatlho P, Muthoga CW, Mutukwa N, Vurayai M, et al. Molecular surveillance of Plasmodium falciparum drug resistance 
markers in clinical samples from Botswana. Am J Trop Med Hyg. 2018;99:1499-503.

23. Talundzic E, Plucinski MM, Biliya S, Silva-Flannery LM, Arguin PM, Halsey ES, et al. Advanced molecular detection of malarone resistance. Antimicrob Agents Chemother. 2016;60:3821-3.

24. Talundzic E, Ravishankar S, Kelley J, Patel D, Plucinski M, Schmedes S, et al. Next-generation sequencing and bioinformatics protocol for malaria drug resistance marker surveillance. Antimicrob Agents Chemother. 2018;62:e02474-517.

25. Menegon M, Nurahmed AM, Talha AA, Nour BY, Severini C. Molecular surveillance of antimalarial drug resistance related genes in Plasmodium falciparum isolates from Eritrea. Acta Trop. 2016;157:158-61.

26. Talundzic E, Maganga M, Masanja IM, Peterson DS, Udhayakumar V, Lucchi NW. Field evaluation of the photo-induced electron transfer fluorogenic primers (PET) real-time PCR for the detection of Plasmodium falciparum in Tanzania. Malar J. 2014;13:31.

27. Mace KE, Arguin PM, Lucchi NW, Tan KR. Malaria surveillance-United States, 2016. MMWR Surveill Summ. 2019;68:1-35.

28. ECDC. European Centre for Disease Control and Prevention. Rapid risk assessment: hospital-acquired malaria infections in the European Union. Stockholm; 2018. https://ecdc.europa.eu/sites/portal/files/
documents/2018-04-30-RRA-Hospital-acquired-Malaria-European-Union -with\%20erratum-1.pdf.

29. Nsanzabana C, Ariey F, Beck HP, Ding XC, Kamau E, Krishna S, et al. Molecular assays for antimalarial drug resistance surveillance: a target product profile. PLoS One. 2018;13:e0204347.

30. WHO. Malaria surveillance, monitoring and evaluation: a reference manual. Geneva: World Health Organization; 2018. https://apps.who.int/ iris/handle/10665/272284.

31. Ocholla H, Preston MD, Mipando M, Jensen AT, Campino S, Maclnnis $B$, et al. Whole-genome scans provide evidence of adaptive evolution in Malawian Plasmodium falciparum isolates. J Infect Dis. 2014;210:1991-2000.

32. Zaw MT, Emran NA, Lin Z. Updates on $\mathrm{k} 13$ mutant alleles for artemisinin resistance in Plasmodium falciparum. J Microbiol Immunol Infect. 2018:51:159-65.

\section{Publisher's Note}

Springer Nature remains neutral with regard to jurisdictional claims in published maps and institutional affiliations. 